\title{
Colecistitis aguda litiásica amebiana como diagnóstico diferencial de cáncer vesicular. Reporte de un caso inusual
}

\author{
Acute amoebic cholecystitis as differential diagnosis of gallbladder cancer. \\ Report of an unusual case
}
Hernán José-Ramírez, ${ }^{*}$ Antonio Palomeque-López, ${ }^{*}$ Néstor Cruz-López, ${ }^{*}$ Yanet Jennings-Aguilar,** Jesús David Guzmán-Ortiz, ${ }^{*}$ Jorge Hernández-Cortez, ${ }^{*}$ Neftalí Cárdenas Herrera***

\footnotetext{
Palabras clave: Cáncer de vesícula biliar, diagnóstico diferencial, amebiasis, amebiasis extraintestinal, absceso hepático amebiano, amebiasis vesicular.

Key words: Gallbladder cancer, differential diagnosis, amebiasis,

extraintestinal amebiasis, amebic

hepatic abscess, gallbladder abscess.
}

* División de Cirugía, Hospital General "Dr. Aurelio Valdivieso", Oaxaca, México.

** División de Ginecología y Obstetricia, Hospital General "Dr. Aurelio Valdivieso", Oaxaca, México.

*** División de Anestesiología Oncológica, Instituto Nacional de Cancerología de México.

Recibido: 29/03/2016 Aceptado: 06/07/2016

\section{RESUMEN}

El cáncer de vesícula biliar es el tumor maligno más común del tracto biliar. Se considera altamente letal. El porcentaje de pacientes diagnosticados tras la colecistectomía simple es de $0.5-1.5 \%$. El diagnóstico diferencial abarca patologías benignas, como procesos inflamatorios e infecciosos a nivel de la vía biliar, incluyendo hidrocolecisto, piocolecisto y colangitis, además de infecciones parasitarias como la infección por E. histolytica (Entamoeba histolytica). La forma de presentación más frecuente de amebiasis es la colitis aguda. Sólo el 10\% de personas infectadas desarrollan síntomas. Las complicaciones se clasifican en intestinales y extraintestinales; entre las intestinales se encuentran hemorragia, megacolon tóxico, apendicitis, ulceraciones graves, perforaciones intestinales y el ameboma. Una pequeña subpoblación desarrolla amebiasis extraintestinal severa. Las manifestaciones extraintestinales incluyen, además del absceso hepático amebiano —que es la más frecuente-, otras poco comunes, como afección pulmonar, cardiaca, cutánea y del SNC, con elevada mortalidad del 4 al 14\%. El absceso hepático amebiano es la manifestación extraintestinal más frecuente; incluso, existe en la literatura el reporte de un caso de absceso esplénico amebiano. La localización en vesícula biliar sólo ha sido documentada en dos ocasiones previamente, y a pesar de considerarse en extremo infrecuente, debe ser tomada en cuenta en el diagnóstico diferencial de cáncer de vesícula biliar.

\section{ABSTRACT}

Gallbladder cancer is the most common malignant tumor of the gastrointestinal tract. It is considered highly lethal. The percentage of patients diagnosed with cancer after a simple cholecystectomy is $0.5-1.5 \%$. The differential diagnosis includes benign conditions such as inflammatory and infectious biliary processes, including hydrocholecyst, pyocholecyst, and cholangitis, as well as parasitic infections such as E. histolytica (Entamoeba histolytica). The most common clinical manifestation of amebiasis is acute colitis. Only $10 \%$ of the subjects infected develop symptoms. The complications classify as intestinal and extraintestinal. Among the intestinal complications are hemorrhage, toxic megacolon, appendicitis, severe ulcerations, intestinal perforations, and ameboma. A small subpopulation develops severe extraintestinal amebiasis. Extraintestinal manifestations include, apart from hepatic abscess, pulmonary, cardiac, cutaneous and NS affections, with an elevated mortality rate ranging from 4 to $14 \%$. Hepatic abscess is the most common extraintestinal manifestation, and there are reports even of an amebic spleen abscess; the finding of an amoebian abscess in the gallbladder has only been reported twice previously, and even though it is considered extremely rare, it must be considered as a differential diagnosis of gallbladder cancer.

\section{INTRODUCCIÓN}

$\mathrm{E}^{1}$ I cáncer vesicular es el más frecuente del tracto biliar. Su forma de presentación puede ser: previo a la cirugía, descubierto durante la colecistectomía y diagnosticado en el examen histopatológico. Al momento del diagnóstico, menos del $20 \%$ de los pacientes son candidatos a cirugía curativa. ${ }^{1}$ En México, en 2003 se reportó un total de 1,052 casos. $^{2}$ El diagnóstico diferencial incluye una gama de patologías benignas, malformaciones estructu- 
rales e, inclusive, infecciones parasitarias del tracto biliodigestivo por $E$. histolytica.

La amebiasis es definida por la Organización Mundial de la Salud como la infección por E. histolytica, protozoario con alta prevalencia en países con condiciones socioeconómicas y sanitarias precarias. Noventa por ciento de las infecciones son asintomáticas y autolimitadas. ${ }^{3}$ Infecta a 500 millones de personas por año, causa enfermedad en 50 millones y se considera la tercera causa de muerte por parasitosis. ${ }^{4}$

En México, es una de las 20 principales causas de enfermedad. ${ }^{5}$ La incidencia de 1995 al año 2000 se reportó entre 1,000 y 5,000 casos por cada 100,000 habitantes al año. ${ }^{6}$ La incidencia del absceso hepático amebiano como complicación extraintestinal más frecuente, fue de 10 casos por 100,000 habitantes al año entre 1995 y el año 2000.

La principal característica del parásito es su gran potencial para invadir y destruir tejidos. Entre los mecanismos de patogenicidad en E. histolytica se encuentra la presencia de la lecitina de galactosa-galactosamina en la superficie de los trofozoítos, responsables de la adhesión a las células intestinales. Las lesiones son caracterizadas por una extensa necrosis, lo que las distingue de otros trastornos causados por diversos microorganismos. ${ }^{7}$ La patogénesis parece resultar de la actividad citotóxica del parásito, que destruye las células huésped en cuestión de minutos. ${ }^{8}$ Estudios han informado de un aumento en la expresión de moléculas de adhesión en las células endoteliales sinusoidales en muestras de abscesos hepáticos, lo que provoca la acumulación de más neutrófilos como respuesta infecciosa e inflamatoria. ${ }^{9}$

La mayoría de las infecciones son asintomáticas; la forma de presentación con síntomas más frecuente de la amebiasis es la colitis amebiana aguda. ${ }^{10}$ El cuadro clínico varía desde diarrea hasta colitis fulminante. El diagnóstico debe basarse en la identificación microscópica de E. histolytica, detección de antígenos específicos o proteína $C$ reactiva (PCR) en heces. ${ }^{11}$ Es bien sabido que en el laboratorio clínico, la prueba más contundente de la culpabilidad amebiana en un caso de disentería es la presencia de amebas hematófagas en heces; es decir, amebas que han ingerido glóbulos rojos del huésped a través del fenómeno de fagocitosis. En la presentación extraintestinal, el hallazgo del parásito en el material necrótico o la determinación de $E$. histolytica por PCR en el aspirado de la lesión son métodos diagnósticos con buenos resultados. ${ }^{12,13}$

Las complicaciones de la enfermedad pueden clasificarse en intestinales y extraintestinales. Entre las complicaciones intestinales se encuentran colitis necrotizante aguda, hemorragia, megacolon tóxico, apendicitis aguda, ulceraciones graves, úlcera perianal con fístulas a este nivel, perforación intestinal y ameboma. ${ }^{14}$ Dependiendo de su forma de presentación, puede ser necesario el tratamiento quirúrgico. La colitis aguda fulminante o necrotizante ocurre alrededor del $0.5 \%$ de los casos; ésta requiere intervención quirúrgica y tiene una mortalidad mayor del $40 \% .{ }^{15}$

Las complicaciones extraintestinales no son habituales e incluyen desde el absceso hepático amebiano hasta manifestaciones poco comunes, como afección pulmonar, cardiaca y del SNC. ${ }^{3}$ El órgano más afectado es el hígado, hasta en el $80 \%$ de los casos. ${ }^{16}$ La respuesta hepática para una invasión amebiana es la inflamación aguda, hiperactivación endotelial y disfunción de la microcirculación que provoca el desarrollo de áreas necróticas. ${ }^{15}$ Pueden surgir complicaciones a partir del absceso hepático, como su ruptura, con diseminación posterior al peritoneo, cavidad pleural o pericardio. ${ }^{14}$ La presencia de abscesos pulmonares sugiere diseminación hematógena; a nivel pleural puede causar empiema, derrame pleural reactivo y fístula broncopleural con hidroneumotórax. ${ }^{17}$

El absceso cerebral de origen amebiano es una forma letal de amebiasis invasiva. ${ }^{18} \mathrm{Se}$ requieren estudios de imagen y pruebas serológicas para su diagnóstico. ${ }^{19}$

La amebiasis cutánea se presenta como úlceras en la región perianal y genital junto con disentería amebiana. ${ }^{20}$ Sin tratamiento, la progresión es implacable, con destrucción masiva de piel y tejido subcutáneo. ${ }^{21}$ Se describen en la literatura cuatro casos de amebiasis en genitales externos, en forma de úlceras solitarias. ${ }^{22}$

Debido a las características de virulencia de E. histolytica, este parásito anaerobio tiene la capacidad de infectar, invadir y destruir prácticamente cualquier tejido del organismo. Se han reportado no sólo casos de amebiasis hepática, 
cutánea, cerebral, pleural, pulmonar, sino que incluso existe el reporte de un caso de absceso esplénico amebiano; ;,23 la localización a nivel de vesícula biliar sólo ha sido documentada previamente en dos ocasiones. ${ }^{24,25}$

En el presente trabajo damos a conocer el caso de amebiasis vesicular en una paciente intervenida quirúrgicamente, con evolución favorable tras su manejo médico y quirúrgico.

Las metas del abordaje para colitis amebiana son tratar la enfermedad invasiva y erradicar la portación intestinal. El metronidazol se considera el fármaco de elección para manejar la amebiasis invasiva. ${ }^{26}$

\section{PRESENTACIÓN DEL CASO}

Paciente femenina de 68 años de edad, hipertensa de larga evolución, en tratamiento, padecimiento de dos años de evolución con dolor en el hipocondrio derecho tipo cólico, irradiado a espalda y hombro ipsilateral. Fue valorada en un servicio médico-quirúrgico, donde se estableció el diagnóstico de colelitiasis crónica por medio de un estudio ecográfico, y fue programada para colecistectomía abierta. Durante el procedimiento, se reportó tumoración dependiente de la vesícula con infiltración al duodeno, estómago, ángulo hepático del colon y epiplón. No se realizó biopsia y fue referida a esta unidad al Servicio de Cirugía Oncológica con el diagnóstico clínico de cáncer de vesícula biliar. Se realizó

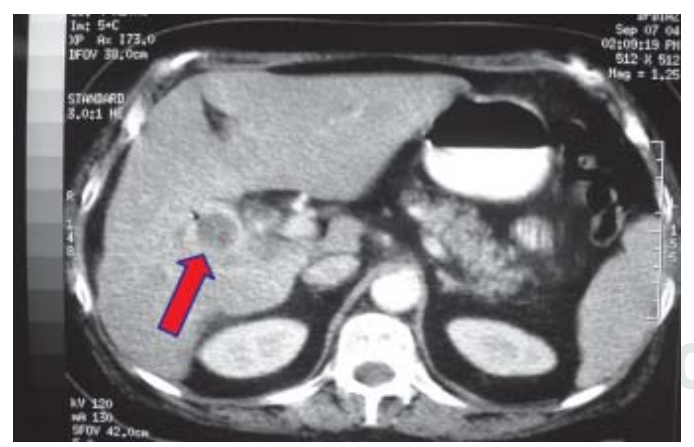

Figura 1. Estudio tomográfico donde se aprecia el gran engrosamiento marcado de la pared vesicular, con un área irregular de la misma de aspecto infiltrante que hizo considerar la posibilidad de cáncer de vesícula (flecha). tomografía computarizada simple y contrastada abdominal, en la cual se observaron cambios postquirúrgicos, vesícula biliar dilatada, su pared libre, ligeramente irregular, con litos en el interior y engrosamiento de hasta $15 \mathrm{~mm}$ de la pared; existió duda de invasión a la pared duodenal (Figura 1). Los estudios de laboratorio prequirúrgicos, incluyendo pruebas de funcionamiento hepático y marcadores tumorales, se encontraron dentro de los límites normales. Se propuso una nueva intervención quirúrgica; la lesión aparentemente era resecable. Se realizó colecistectomía radical; se identificó una intensa reacción inflamatoria pericolecística con adherencias de epiplón a la pared abdominal, vesícula con litos en su interior, necrosis de la pared del cuerpo, íntimamente adherida a la pared duodenal a nivel de la segunda porción, sin presencia de fistulización; no se observó infiltrado a hígado u otros órganos (Figura 2).

El estudio histopatológico del producto de la colecistectomía radical reportó una vesícula biliar con dimensiones de 5 × $3.5 \times 3.5 \mathrm{~cm}$; la superficie externa, grisácea anfractuosa; al corte, el espesor de su pared variaba de 0.3 a $2 \mathrm{~cm}$, con necrosis panmural segmentaria y parchada; la mucosa era rojiza, parcialmente ulcerada; el conducto cístico se encontró permeable; no se identificó ganglio cístico. En el estudio microscópico de los cortes se identificaron trofozoítos de E. histolytica con eritrofagocitosis en todo el espesor de la pared, incluyendo submucosa, muscular y subserosa, rodeados de proceso inflamatorio agudo con áreas de necrosis y de un proceso inflamatorio de polimorfonucleares moderado (Figuras 3 a 5). Además, se reportó un

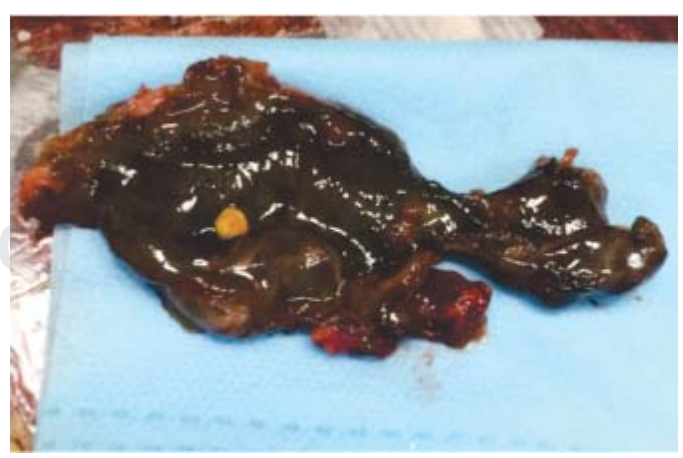

Figura 2. Pieza quirúrgica producto de colecistectomía radical. 


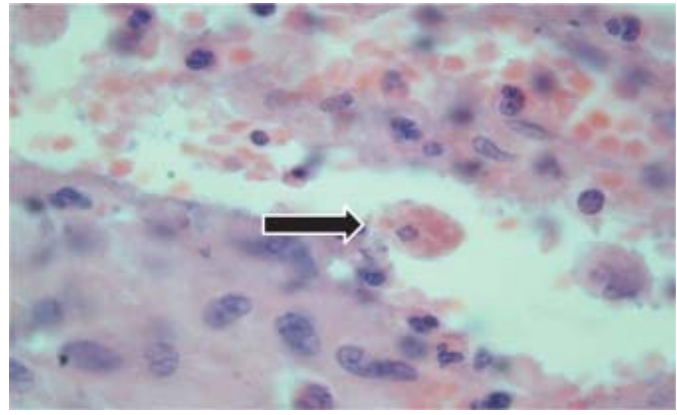

Figura 3. Microfotografía donde se aprecia eritrofagocitosis por trofozoítos y la presencia de mononucleares y algunas células de la mucosa vesicular.

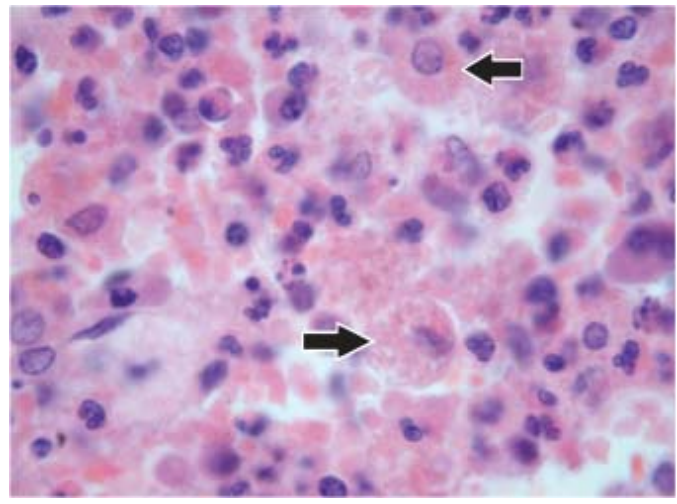

Figura 4. Microfotografía donde se aprecia intenso infiltrado inflamatorio por polimorfonucleares y la presencia de trofozoitos con eritrofagocitosis caracteristicas de E. histolytica (flechas).

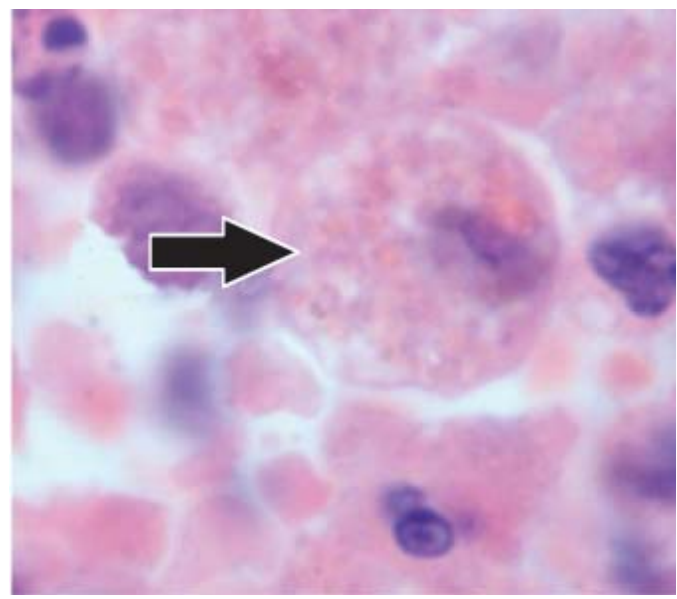

Figura 5. Aumento de la microfotografía anterior, donde se aprecia a mayor detalle el trofozoito de E. histolytica (flecha). lito único de $2.5 \mathrm{~mm}$ de diámetro de cristales de colesterol, segmentectomía hepática con inflamación crónica ligera en espacio porta, negativo a malignidad. Tejido pericolecístico con inflamación aguda y crónica.

Se inició un tratamiento con metronidazol y fue valorada por el Servicio de Infectología, quien continuó con mismo manejo a dosis de 750 mg tres veces al día por 10 días.

La evolución posterior fue satisfactoria, y en el estudio coproparasitoscópico posterior no se identificaron parásitos de E. histolytica.

\section{DISCUSIÓN}

El cáncer de vesícula biliar es la neoplasia más frecuente del árbol biliar. Es una causa importante de mortalidad. El diagnóstico se establece, en la mayoría de las ocasiones, en etapas avanzadas, cuando existe invasión a órganos contiguos y enfermedad metastásica, o bien, se identifica de forma incidental durante una colecistectomía por litiasis vesicular o, en ocasiones, en el estudio histológico de la vesícula posterior a una colecistectomía por litiasis vesicular. Debido al número reducido de casos sospechosos de malignidad previos a la cirugía curativa, el diagnóstico diferencial debe ser exhaustivo y deberá incluir patologías o lesiones benignas, tales como litiasis vesicular, colangitis, piocolecisto, vesícula en porcelana, malformaciones estructurales del árbol biliar; inclusive, actualmente se recomienda poner énfasis en procesos infecciosos parasitarios como el comentado en el presente caso.

E. histolytica es el agente causal de la amebiasis invasiva; es endémica en regiones con condiciones sanitarias deficientes, como nuestro país. Las formas quísticas se expulsan con las heces y son resistentes a las condiciones ambientales; la transmisión es fecal-oral. Aunque no suele ser posible hacer la distinción etiológica con base en la presentación clínica de la enfermedad, se debe considerar como agente causal de amebiasis invasiva y causante de amebiasis extraintestinal.

Encontramos este caso de particular interés debido a la forma rara de presentación. En su manifestación clínica, Ilama la atención la ausencia total de antecedentes de disentería amebiana o colitis amebiana como evento 
previo, así como la ausencia de abscesos amebianos a nivel de otras estructuras orgánicas en su evolución; sin embargo, podría tratarse de una paciente cuya infección parasitaria no precisó atención alguna en sus inicios. Es importante mencionar que las complicaciones extraintestinales de la amebiasis se presentan, por lo regular, con manifestaciones clínicas de una infección severa, ya sea la formación de un absceso o fístulas a cualquier nivel; en el presente caso, no existió ninguna de estas manifestaciones.

Una vez realizada una extensa revisión bibliográfica sobre amebiasis extraintestinal, sólo se encontraron dos publicaciones que documentan la infección por el protozoario a nivel de la vesícula biliar. El primero, en 1934, en Egipto, en una revisión de nueve casos de parasitosis de vesícula, entre los cuales se describe un caso de E. histolytica identificado en el estudio post mortem de un paciente que falleció por disentería amebiana. La segunda publicación proviene de España, en 1990, por Fernández y colaboradores, ${ }^{24,25}$ donde se describe un caso diagnosticado como colecistitis aguda alitiásica tratada con colecistectomía; en el estudio histológico se identifican los quistes de E. histolytica en la pared de la vesícula.

Sin duda alguna, la amebiasis vesicular es una afección muy rara. Debido a su extrema singularidad, no existe descripción fisiopatológica de la afección de vesícula biliar por un parásito de origen intestinal; sin embargo, consideramos que debe ser similar a la afección del hígado, ampliamente descrita en múltiples publicaciones. La forma de diseminación es por vía venosa portal a través de vasos comunes que se comparten con el lecho vesicular, la invasión a la mucosa vesicular con destrucción y necrosis de la misma, y finalmente, la invasión al resto de la pared. Sin duda alguna, E. histolytica cuenta con mecanismos de adhesión celular altamente efectivos para tener la capacidad de afectar prácticamente cualquier órgano.

El caso que se reporta es posiblemente el primero del cual tenemos noticia en nuestro país y Latinoamérica, y el tercero publicado en la literatura mundial. Por ende, y a pesar de que la amebiasis vesicular es en extremo infrecuente, debe considerarse como un diagnóstico diferencial remoto del cáncer de vesícula biliar, y más aún si se tiene el antecedente clínico de disentería. Sin embargo, consideramos que, desgraciadamente, sólo se llega a este diagnóstico por medio del estudio histológico de la pieza quirúrgica, como ocurrió en el presente caso.

\section{REFERENCIAS}

1. Kanthan R, Senger JL, Ahmed S, Kanthan SC. Gallbladder cancer in the 21st century. J Oncol. 2015; 2015: 967472. doi: 10.1155/2015/967472. Epub 2015 Sep 1.

2. Padilla-Rosciano AE, Cruz-Rodríguez A, Serna-Thomé MG, Calderillo-Ruiz G, Vela-Sarmiento I, LópezBasave HN, et al. Cáncer de vesícula y vías biliares. Manual de Oncología: procedimientos médico quirúrgicos. 5. ${ }^{\text {a }}$ edición. McGraw Hill; 2013.

3. Sateriale A, Huston CD. A sequential model of host cell killing and phagocytosis by Entamoeba histolytica. J Parasitol Res. 2011; 2011: 926706. doi: 10.1155/2011/926706.

4. Entamoeba taxonomy. Bull World Health Organ. 1997; 75: 291-294

5. Gil-Manrique LF, Soler-Morejón C, Crespo-Acebal L, Villa-Valdés M, Alfonso-Alfonso L, Contreras-Roja IC. Absceso esplénico amebiano. Presentación de un caso. Revista Cubana de Cirugía. 2011; 50: 560-569.

6. Ximénez C. Epidemiology of amebiasis in Mexico: a molecular approach. Arch Med Res. 2006; 37: 263265.

7. Caballer-Salcedo A, Vivero-Rogel M, Salvatierra B, et al. Seroepidemiology of amebiasis in Mexico. Am J Trop Med Hyg. 1994; 50: 412-419.

8. Aguirre-García M, Gutiérrez-Kobeh L, López-Vancell R. Entamoeba histolytica: adhesins and lectins in thetrophozoite surface. Molecules. 2015; 20: 2802-2815.

9. Campos-Rodríguez R, Jarillo-Luna RA, Larsen BA, Rivera-Aguilar V, Ventura-Juárez J. Invasive amebiasis: a microcirculatory disorder? Med Hypotheses. 2009; 73: 687-697.

10. Roca-Goderich R. Enfermedad diarreica aguda. Amebiasis intestinal. En: Temas de medicina interna. Tomo III. 4. ${ }^{\text {a }}$ ed. La Habana: Editorial Ciencias Médicas; 2002. p. 526-529.

11. Salles JM, Salles MJ, Moraes LA, Silva MC. Invasive amebiasis: an update on diagnosis and management. Expert Rev Anti Infect Ther. 2007; 5: 893-901.

12. Patricio C, Amaral P, Lourenço J. An uncommon case of hepatopulmonary amoebiasis. BMJ Case Rep. 2014; 2014. pii: bcr2014204129. doi: 10.1136/bcr-2014204129.

13. Kunst H, Mack D, Kon OM, Banerjee AK, Chiodini P, Grant A. Parasitic infections of the lung: a guide for the respiratory physician. Thorax. 2011; 66: 528-536. doi: $10.1136 /$ thx.2009.132217.

14. Haque R, Huston CD, Hughes M, Houpt E, Petri WA Jr. Amebiasis. N Engl J Med. 2003; 348: 1565-1573.

15. Pérez-Tamayo R, Montfort I, García AO, Ramos E, Ostria CB. Pathogenesis of acute experimental liver amebiasis. Arch Med Res. 2006; 37: 203-209.

16. Fang D, Shu D. Entamoeba histolytica liver abscess. CMAJ. 2010; 182: 1758. 
17. Lal C, Huggins JT, Sahn SA. Parasitic diseases of the pleura. Am J Med Sci. 2013; 345: 385-389. doi: 10.1097/MAJ.0b013e318266e984.

18. Maldonado-Barrera CA, Campos-Esparza MR, Muñoz-Fernández L, Victoria-Hernández JA, CamposRodríguez R, Talamás-Rohana P, et al. Clinical case of cerebral amebiasis caused by E. histolytica. Parasitol Res. 2012; 110: 1291-1296. doi: 10.1007/s00436011-2617-8. Epub 2011 Aug 26.

19. Abdel Razek AA, Watcharakorn A, Castillo M. Parasitic diseases of the central nervous system. Neuroimaging Clin N Am. 2011; 21: 815-841, viii. doi: 10.1016/j. nic.2011.07.005.

20. Magaña ML, Fernández-Díez J, Magaña M. Cutaneous amebiasis in pediatrics. Arch Dermatol. 2008; 144: 1369-1372. doi: 10.1001/archderm.144.10.1369.

21. Fernández-Díez J, Magaña M, Magaña ML. Cutaneous amebiasis: 50 years of experience. Cutis. 2012; 90: 310-314.

22. Abdolrasouli A, de Vries HJ, Hemmati $Y$, Roushan A, Hart J, Waugh MA. Sexually transmitted penile amoebiasis in Iran: a case series. Sex Transm Infect. 2012; 88: 585-588. doi: 10.1136/sextrans- 2012 050577. Epub 2012 Aug 28.

23. Fonseca Aizpuru EM, Nuño Mateo FJ, Menéndez Calderon MJ, Arias Miranda IM, Rodríguez Díaz B.
Splenic abscess: revision of 7 cases. An Med Interna (Madrid). 2007; 24: 511-512.

24. Hashim M. Pathological lesions of the gall-bladder associated with ulcerations and bilharziasis of the intestine. A case of amoebic dysentery in which the morbid histology of the gallbladder has revealed E.histolytica in its wall is recorded and the literature on "amoebic cholecystitis" is reviewed. Transactions of the Royal Society of Tropical Medicine and Hygiene. 1934; 27: 399-407.

25. Fernández FAH, Robles CS, Carratalá FB, Espejo JM, Moreno FP. Colecistitis aguda alitiásica amebiana. Revista Española de las Enfermedades del Aparato Digestivo. 1990; 78: 41-42

26. Antiamoebic drugs for treating amoebic colitis. Cochrane Database of Systematic Reviews. The Cochrane Library, Issue 2, Art. No. CD006085. Cochrane Database of Systematic Reviews, Issue 2, 2015.

Correspondencia:

Dr. Hernán José-Ramírez

Porfirio Díaz Núm. 400,

Colonia Reforma, 68050,

Oaxaca de Juárez, Oaxaca, México.

Tel: 9515151300

E-mail: hernanjr36@hotmail.com 